\title{
Digital Medicine in Thyroidology: A New Era of Managing Thyroid Disease
}

\author{
Jae Hoon Moon ${ }^{1}$, Steven R. Steinhubl ${ }^{2}$ \\ ${ }^{1}$ Department of Internal Medicine, Seoul National University Bundang Hospital, Seoul National University College of \\ Medicine, Seongnam, Korea; ${ }^{2}$ Department of Molecular Medicine, Scripps Research Translational Institute, La Jolla, CA, USA
}

Digital medicine has the capacity to affect all aspects of medicine, including disease prediction, prevention, diagnosis, treatment, and post-treatment management. In the field of thyroidology, researchers are also investigating potential applications of digital technology for the thyroid disease. Recent studies using artificial intelligence (AI)/machine learning (ML) have reported reasonable performance for the classification of thyroid nodules based on ultrasonographic (US) images. AI/ML-based methods have also shown good diagnostic accuracy for distinguishing between benign and malignant thyroid lesions based on cytopathologic findings. Assistance from AI/ML methods could overcome the limitations of conventional thyroid US and fine-needle aspiration cytology. A webbased database has been developed for thyroid cancer care. In addition to its role as a nationwide registry of thyroid cancer, it is expected to serve as a clinical platform to facilitate better thyroid cancer care and as a research platform providing comprehensive disease-specific big data. Evidence has been found that biosignal monitoring with wearable devices may predict thyroid dysfunction. This real-world thyroid function monitoring could aid in the management and early detection of thyroid dysfunction. In the thyroidology field, research involving the range of digital medicine technologies and their clinical applications is expected to be even more active in the future.

Keywords: Thyroid; Thyroid neoplasms; Hyperthyroidism; Hypothyroidism; Artificial intelligence; Machine learning; Database; Wearable electronic devices

\section{INTRODUCTION}

Digital medicine has become one of the hottest topics in medicine over the last decade. Several conferences, forums, and academic associations and journals focusing on digital medicine or digital healthcare have been established, and the global digital medicine market is expected to reach over 500 billion United States dollar by the end of 2025, growing at a compound annual growth rate of $30 \%$ [1]. "Digitization" of medical information began a long time ago with the introduction of computers. Various pieces of information measured from patients, such as height, weight, blood pressure, pulse rate, and blood test results,
Received: 20 May 2019, Revised: 23 May 2019, Accepted: 27 May 2019

Corresponding authors: Jae Hoon Moon

Division of Endocrinology and Metabolism, Department of Internal Medicine, Seoul National University Bundang Hospital, Seoul National University College of Medicine, 82 Gumi-ro 173beon-gil, Bundang-gu, Seongnam 13620, Korea Tel: +82-31-787-7068, Fax: +82-31-787-4052, E-mail: jaemoon76@snubh.org

Steven R. Steinhubl

Department of Molecular Medicine, Scripps Research Translational Institute, 3344 North Torrey Pines Ct, Plaza Level, La Jolla, CA 92037, USA

Tel: +1-858-784-2184, Fax: +1-570-271-8005, E-mail: steinhub@scripps.edu

\section{Copyright $\odot 2019$ Korean Endocrine Society}

This is an Open Access article distributed under the terms of the Creative Commons Attribution Non-Commercial License (http://creativecommons.org/ licenses/by-nc/4.0/) which permits unrestricted non-commercial use, distribution, and reproduction in any medium, provided the original work is properly cited. 
have been stored and managed in databases. The introduction of picture archiving and communication systems and electronic health records (EHRs) made it possible to store digitized medical images and records in databases, to access them using multiple modalities, and to transmit them via networks. Based on these sources of digitized medical information, remarkable recent developments in information and communication technology, biosensor technology, and artificial intelligence (AI)/machine learning (ML) technology have caused digital medicine to emerge as a highly promising field from both academic and industrial perspectives.

Digital medicine not only makes it possible to use digital devices for medical purposes, but also changes the overall landscape of disease prediction, prevention, diagnosis, treatment, and post-treatment management. Furthermore, it changes the ways in which medical providers and consumers communicate, use medical resources, and bill and pay for medical expenses. Digital medicine has also affected the field of thyroidology, as researchers have investigated potential ways to use digital technology for the diagnosis, treatment, and prevention of thyroid disease. In this review, we discuss recent research and clinical applications of digital medicine in the field of thyroidology.

\section{IMAGING ANALYSIS USING AI/ML}

The rapid growth of computer technology has paved the way for the development of innovative technologies that transcend human capabilities in certain areas, as exemplified by AI and ML. Broadly speaking, AI can be understood as the use of machines to mimic human cognitive function, including learning and problem-solving. Academically, AI is the study of intelligent agents that can recognize the complexity of the environment and maximize the possibility of achieving their goals [2]. $\mathrm{ML}$ is a field of AI that researches the algorithms and statistical models that enable computer systems to improve their performance on a specific task through experience without being explicitly programmed [3]. In the medical field, AI/ML has been most actively applied to the analysis of medical images. AI/ML methods can interpret digitized medical images accurately, providing information that physicians can use to make a computeraided diagnosis (CAD). In the field of thyroidology, most studies of AI/ML have focused on the differentiation of benign and malignant thyroid nodules in ultrasonographic (US) images. US is the primary imaging modality used to examine thyroid nodules [4], and significant research has sought to predict the malignant potential of thyroid nodules based on US features [5-7].
However, conventional US is operator-dependent and its diagnostic outcomes are affected by the experience, knowledge, and labor intensity of radiologists [8]. For this reason, AI/ML methods have been applied with the aim of assisting or automating the differential diagnosis of thyroid nodules. In recent years, several studies have been conducted to develop a CAD system using ML technology for classifying thyroid nodules in US images, using approaches that can be categorized into hand-crafted feature-based classifiers and data-driven methods [9,10].

Hand-crafted feature-based classifiers use features extracted from US images by researchers. Although these features can be clinical or non-clinical, most ML classifiers use non-clinical features for reasons described later. As a basic concept, handcrafted feature-based classifiers are similar to the conventional classification methods used by radiologists, who make diagnostic decisions based on a combination of their accumulated experience, expertise, and cognitive acumen with clinical features from US images such as microcalcification, margins, shape, echogenicity, contents, and vascularity. ML classifiers use a combination of ML algorithms and non-clinical features from US images such as textural features, vascular features, and discrete wavelet transform features [9]. Through the supervised learning process, ML classifiers accomplish their tasks of identifying thyroid nodules, classifying and estimating their malignancy risk, and differentiating types of malignancies [11]. In their review, Acharya et al. [9] claimed that ML classifiers using non-clinical features showed higher classification accuracies than were obtained by radiologists using clinical features, and also presented the following additional advantages of using non-clinical features: (1) such features can be automatically extracted from US images and quantified as numerical values that can be used for automated classification; (2) there is no need for visual inspection and interpretation, and therefore, the results are more objective; and (3) such CAD systems can be written as software applications at a low cost and installed on existing computers in clinics at no extra cost. The accuracy of ML classifiers for thyroid nodule classification in recently published studies is shown in Table 1 [12-17].

Data-driven learning methods do not need hand-crafted features. Convolutional neural networks $(\mathrm{CNNs})$ can extract features from natural images, thereby improving the classification and detection performance in image analysis. Ma et al. [18] were the first researchers to apply CNNs to the task of thyroid nodule diagnosis. They reported an accuracy of $83.02 \% \pm 0.72 \%$ for thyroid nodule classification (benign vs. malignant) and proposed potential clinical applications of $\mathrm{CNN}$-based methods. 


\begin{tabular}{|c|c|c|c|c|}
\hline Study & Subjects & Features & Classifier & Accuracy, \% \\
\hline Acharya et al. (2011) [12] & 10 Benign, 10 malignant & Texture and DWT features from CEUS images & $\mathrm{kNN}$ & 98.9 \\
\hline Ding et al. (2011) [13] & 69 Benign, 56 malignant & Statistical and textural features & SVM & 93.6 \\
\hline Acharya et al. (2012) [14] & 10 Benign, 10 malignant & Texture and DWT features & AdaBoost & 100 \\
\hline Acharya et al. (2012) [15] & 10 Benign, 10 malignant & $\begin{array}{l}\text { Fractal dimension, fourier spectrum descriptor, } \\
\text { local binary patterns, laws texture energy } \\
\text { from 3-D HRUS and CEUS images }\end{array}$ & $\begin{array}{l}\text { Gaussian mixture } \\
\text { model, fuzzy, SVM }\end{array}$ & $98.1-100$ \\
\hline Chang et al. (2016) [16] & 29 Benign, 30 malignant & Texture features & SVM & 98.3 \\
\hline Prochazka et al. (2019) [17] & 20 Benign, 20 malignant & Histogram features, SFTA & SVM, RF & 94.64 \\
\hline
\end{tabular}

Ko et al. [19] recently reported that the areas under the curve (AUCs) for diagnosing thyroid malignancy were 0.835 to 0.850 for three CNNs, and stated that these results were comparable to those of radiologists (AUC, 0.805 to 0.860 ). In the aforementioned studies, radiologists were required to delineate the boundary of thyroid nodules [18] or to draw square regions of interest covering thyroid nodules [19] in each image. In a recent study by Wang et al. [20], the YOLOv2 neural network was used to detect and classify benign and malignant thyroid nodules, and the AI system successfully identified the lesion areas as well as its diagnostic performance was not significantly different compared with radiologists.

Current research into imaging analysis has reported reasonable performance for the use of AI/ML to assist clinicians in classifying thyroid nodules using US images. These AI/ML methods are expected to compensate for the weaknesses of the operator-dependent and labor-intensive diagnostic process based on manual US. Considering their advantages compared to handcrafted feature-based classifiers, data-driven learning methods are likely to be at the forefront of future research into the clinical applications of AI/ML for thyroid nodule classification.

\section{CYTOPATHOLOGIC ANALYSIS USING AI/ML}

When US images are suspicious for a thyroid malignancy, fineneedle aspiration (FNA) is recommended [4]. FNA is relatively simple, minimally invasive, painless, inexpensive, and highly accurate. Cytopathologists examine FNA specimens under microscopy and make decisions based on their knowledge and experience. Therefore, FNA cytology-based diagnoses are subjective and prone to inter-observer variability. To overcome these problems, several mathematical and computational models have been applied for the diagnostic cytology of thyroid nodules, with reasonable accuracy [21-30]. AI/ML methods were first applied to the analysis of thyroid FNA specimens by Karakitsos et al. [21] in 1996. They used 26 features describing the size, shape, and texture of the nucleus with an artificial neural network (ANN) model in 51 cases and reported a correct classification of $90.6 \%$ of nuclei, including cases of follicular adenoma, follicular carcinoma, papillary carcinoma, and Hashimoto thyroiditis. AI/ML methods could be valuable in cases with indeterminate findings on cytology. Ippolito et al. [23] studied 453 patients with a thyroid nodule diagnosed with indeterminate findings on FNA biopsy using an ANN analysis with cytologic and clinical data. They categorized the patients from high to low risk, and found that the ANN model showed higher sensitivity and specificity for distinguishing between benign and malignant nodules than the standard cytologic criteria. AI/ML methods also could be applied to the differentiation of follicular neoplasms, for which benign and malignant lesions cannot be discriminated using traditional cytologic diagnostic techniques. Shapiro et al. [24] reported a diagnostic accuracy of $87 \%$ for distinguishing follicular adenomas from carcinomas with an ANN method using nuclear morphometric parameters and density features of chromatin texture. $\mathrm{CNN}$, which is a data-driven learning method, was recently investigated as a way to distinguish papillary thyroid carcinoma (PTC) from non-PTC (colloid goiter, cytologically diagnosed follicular neoplasms, and lymphocytic thyroiditis) [29]. In that study, 370 images of $512 \times 512$ pixels (186 PTC and 184 non-PTC) photographed from 20 cytology smears (from 20 patients) were used to train the software. Although the CNN model showed a diagnostic accuracy of 
$85.06 \%$, the gold standard of diagnosis was cytologic diagnosis, not pathologic diagnosis, and the CNN model was only used to distinguish PTC from non-PTC thyroid lesions, not to differentiate other categories of thyroid lesions.

AI/ML-based methods for distinguishing benign and malignant thyroid lesions based on cytopathologic findings have shown good diagnostic accuracy, and they may assist cytopathologists in their decision-making. Furthermore, AI/ML methods may help overcome the limits of traditional cytopathology in differentiating thyroid follicular neoplasms and lesions showing indeterminate cytology. Nonetheless, advances in image analysis and intensive testing on large data sets of cytopathologic findings are required for the full-scale clinical application of these methods.

\section{DATABASE FOR THYROID DISEASE}

With the efficient management and systematic analysis of medical big data using information and communication technology, web-based platforms have been developed for collecting, standardizing, and storing disease-related data at the national or global level. Using such platforms, patients and physicians can access integrated medical records without temporal or geographical constraints, and researchers can easily analyze highquality, real-world big data. The best example of this phenomenon is provided by cancer care, as clinics and hospitals generate huge amounts of data in EHRs, and big data systems are able to combine those data with findings from the published literature using algorithms to provide better management guidelines [31]. Recently, a web-based database platform for thyroid cancer management was developed, known as the Thyroid Cancer Care Collaborative (TCCC, https://www.thyroidccc.org) [32,33]. The TCCC is a patient-oriented platform. Each thyroid cancer patient can have a TCCC profile, and his or her clinician can report and store all data related to a patient's thyroid cancer care across the following nine distinct modules: initial presentation, imaging, surgical management, postoperative hospital course, postsurgical follow-up/treatment surveillance, pathology and staging, laboratory results, nuclear medicine, and change in health status. This platform provides a number of features for patient education, clinical decision-making, and enhanced cancer care communication, which will benefit patients and clinicians. Mehra et al. [33] introduced the TCCC in a review and claimed that integration of the TCCC and EHRs using a universal language is under development and that the web-based program meets the strictest standards for data encryption and site- wide security at the browser and server level. In addition, the TCCC informs patients about the use of their data for research, and informed consent for research can be obtained online. Since multidisciplinary management over a long time period is required for the treatment of thyroid cancer, this web-based platform can be a solution for maintaining comprehensive data on patients despite changes in their geographical location or physicians. The TCCC is expected to serve not only as a national registry of thyroid cancer, but also as a clinical platform for better thyroid cancer care and as a research platform providing comprehensive disease-specific big data.

\section{WEARABLES AND THYROID DISEASE}

During recent years, a variety of wearable devices that can monitor various biosignals have been developed for use in hospital environments, as well as in everyday life [34]. The market of wearable activity trackers has grown considerably in recent years, and forecasts predict that sales of wearable activity trackers will increase in the future. Currently available commercial smartbands or smartwatches can monitor the user's activity, sleep, heart rate (HR) and rhythm, electrocardiogram, and oxygen saturation, and various sensors for other biosignals can be integrated with these devices. Several studies have investigated medical applications of the biosignal-monitoring ability of activity trackers, but only one study has done so with a focus on thyroidology. Lee et al. [35] reported the clinical feasibility of monitoring resting HR using a wearable activity tracker in patients with thyrotoxicosis. In their study, they used Fitbit ${ }^{\mathrm{TM}}$ devices (Fitbit, San Francisco, CA, USA), which can measure HR and physical activity using photoplethysmography and a 3-axis accelerometer, respectively. Photoplethysmography measures the differential reflection of light from the skin, based on the pulsatility of superficial blood vessels [36], and a number of studies on the accuracy of these wrist-worn HR monitors have been published, showing relatively accurate resting $\operatorname{HR}[37,38]$. Lee et al. [35] extracted resting HR from the continuously monitored HR and activity data of thyrotoxic patients during their anti-thyroid drug treatment period. In that study, the resting HR from a wearable device was higher in thyrotoxic patients than in the control groups and decreased in association with thyrotoxicosis improvement. An increase in resting HR of approximately 11 beats per minute measured using a wearable device was associated with a $0.5 \mathrm{ng} / \mathrm{dL}$ increase in serum free thyroxine levels and a 3.8 times higher risk of thyrotoxicosis. The authors claimed that monitoring thyroid function using wearable devic- 
es can aid in the management of thyrotoxicosis and early detection of disease recurrence in patients who are in remission after treatment. They also stated that they expected their study to be applicable for preventing overtreatment of levothyroxine replacement in hypothyroid patients. The research group that conducted this study has developed a web-based application to predict thyrotoxicosis using resting HR data from wearable devices (https://thyroscope.org) (Fig. 1) [39].

\section{CONCLUSIONS}

Throughout human history, medicine has always evolved with the adoption of new technologies. Stethoscopes, electrocardiography, X-rays, US, computed tomography, and magnetic resonance imaging likewise resulted from applying the latest technology of the time to medicine, and doing so has led to remarkable advances in medicine. Digital medicine reflects another in-
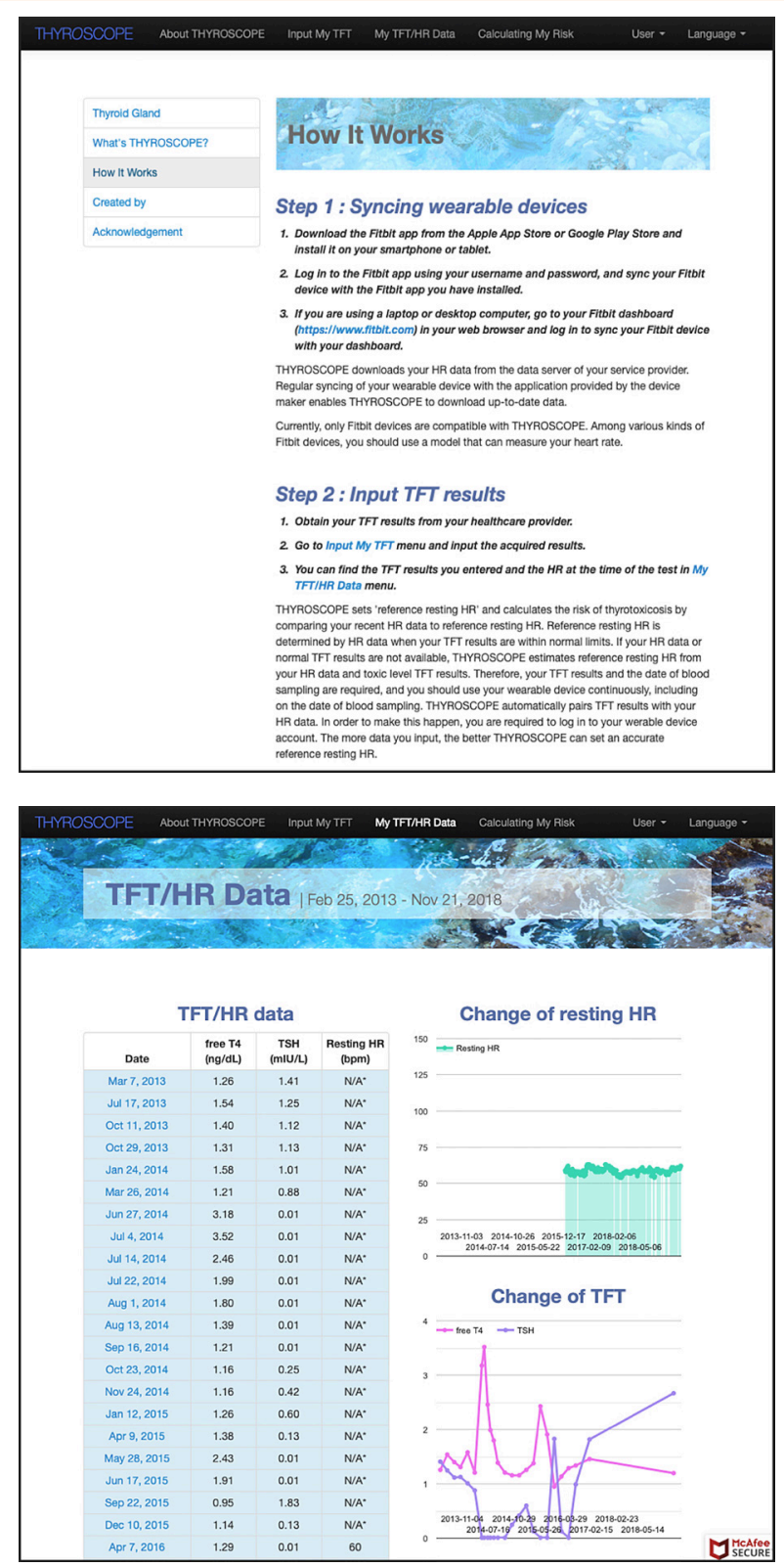
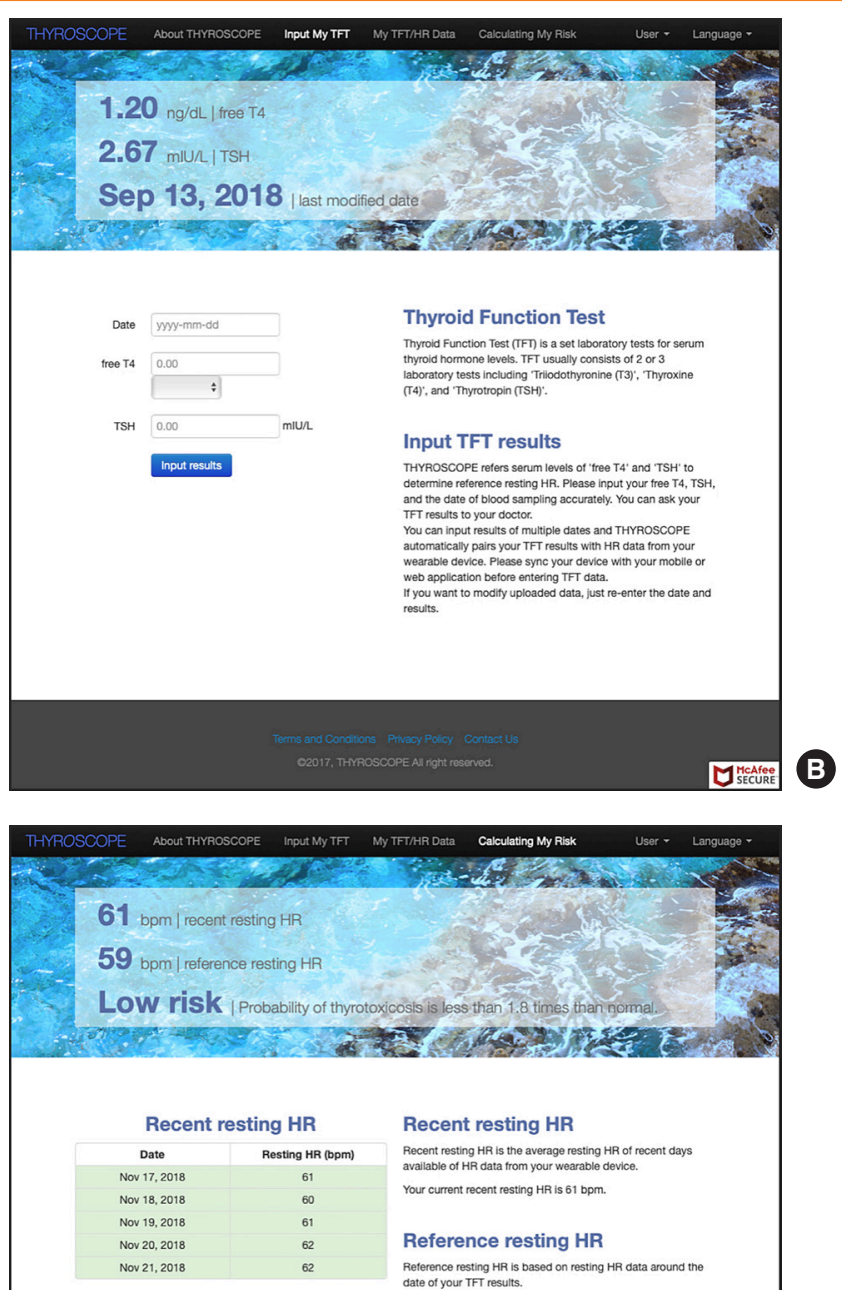

Recent resting $\mathrm{HR}$

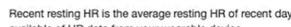

Your current recent resting HR is 61 bpm.

Reference resting HR

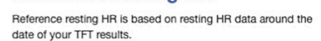

Your current reterence resting HR is $59 \mathrm{bpm}$.

"NA' move.

Reference vs Recent resting HR

Risk level for thyrotoxicosis Aclinic vistit is recommended to subjects with moderate or

- Low risk: Ourrent probability ot thyrotoxicosis is liss than
1.8 times compared to when your thyrold tunction was normal.
- Moderate nisk: Current probability of thyrotoxicosis is 1.8 . 2.9 times than norm
- High nisk: Current
3itmes

Fig. 1. Representative screen shots of a web-based application for predicting the risk of thyrotoxicosis using wearable devices (https:// thyroscope.org). There are four distinct modules: (A) about THYROSCOPE, (B) Input My TFT, (C) My TFT/HR Data, and (D) Calculating My Risk. Reprinted from THYROSCOPE, with permission from THYROSCOPE [39]. TFT, thyroid function test; HR, heart rate. 
stance of new technologies being introduced, but with the difference that it can be more patient-focused and has the potential to affect the entire field of medicine in the future. Although digital medicine is in a very early phase and surrounded by many uncertainties, such as concerns about privacy, security, and costeffectiveness, it already has affected the thyroidology field as described above. We recommend that thyroid specialists should engage with this emerging medical field and apply it in their practice, research, and education to contribute to patient health and wellness.

\section{CONFLICTS OF INTEREST}

Jae Hoon Moon has created and is currently operating the web application, https://thyroscope.org. Otherwise, no potential conflict of interest relevant to this article was reported.

\section{ACKNOWLEDGMENTS}

Steven R. Steinhubl is supported in part by the U.S. National Institutes of Health/National Center for Advancing Translational Sciences grant UL1TR001114.

\section{ORCID}

Jae Hoon Moon https://orcid.org/0000-0001-6327-0575

Steven R. Steinhubl https://orcid.org/0000-0002-9256-7914

\section{REFERENCES}

1. Global Market Insights. Digital Health Market Size By Technology [Tele-healthcare \{Telecare (Activity Monitoring, Remote Medication Management), Telehealth (LTC Monitoring, Video Consultation)\}, mHealth \{Wearables (BP Monitors, Glucose Meter, Pulse Oximeter, Sleep Apnea Monitors, Neurological Monitors), Apps (Medical, Fitness)\}, Health Analytics, Digital Health System (EHR, e-prescribing System)], Industry Analysis Report, Regional Outlook (U.S., Canada, Germany, UK, France, Spain, Italy, Russia, Poland, China, India, Japan, Australia, Brazil, Mexico, South Africa), Application Potential, Price Trends, Competitive Market Share \& Forecast, 2019-2025 [Internet]. Digital Health Market Report 2019. Selbyville: Global Market Insights, Inc.; 2019 [cited 2019 May 26]. Available from: https://www. gminsights.com/industry-analysis/digital-health-market.

2. Poole DL, Mackworth AK, Goebel R. Computational intel- ligence: a logical approach. New York: Oxford University Press; 1998. p. 558.

3. Bishop CM. Pattern recognition and machine learning. New York: Springer; 2006. p. 738.

4. Haugen BR, Alexander EK, Bible KC, Doherty GM, Mandel SJ, Nikiforov YE, et al. 2015 American Thyroid Association Management guidelines for adult patients with thyroid nodules and differentiated thyroid cancer: the American Thyroid Association guidelines task force on thyroid nodules and differentiated thyroid cancer. Thyroid 2016;26:1-133.

5. Smith-Bindman R, Lebda P, Feldstein VA, Sellami D, Goldstein $\mathrm{RB}$, Brasic N, et al. Risk of thyroid cancer based on thyroid ultrasound imaging characteristics: results of a population-based study. JAMA Intern Med 2013;173:1788-96.

6. Brito JP, Gionfriddo MR, Al Nofal A, Boehmer KR, Leppin $\mathrm{AL}$, Reading $\mathrm{C}$, et al. The accuracy of thyroid nodule ultrasound to predict thyroid cancer: systematic review and meta-analysis. J Clin Endocrinol Metab 2014;99:1253-63.

7. Shin JH, Baek JH, Chung J, Ha EJ, Kim JH, Lee YH, et al. Ultrasonography diagnosis and imaging-based management of thyroid nodules: revised Korean Society of Thyroid Radiology consensus statement and recommendations. Korean J Radiol 2016;17:370-95.

8. Park CS, Kim SH, Jung SL, Kang BJ, Kim JY, Choi JJ, et al. Observer variability in the sonographic evaluation of thyroid nodules. J Clin Ultrasound 2010;38:287-93.

9. Acharya UR, Swapna G, Sree SV, Molinari F, Gupta S, Bardales RH, et al. A review on ultrasound-based thyroid cancer tissue characterization and automated classification. Technol Cancer Res Treat 2014;13:289-301.

10. Song W, Li S, Liu J, Qin H, Zhang B, Zhang S, et al. Multitask cascade convolution neural networks for automatic thyroid nodule detection and recognition. IEEE J Biomed Health Inform 2019;23:1215-24.

11. Chi J, Walia E, Babyn P, Wang J, Groot G, Eramian M. Thyroid nodule classification in ultrasound images by fine-tuning deep convolutional neural network. J Digit Imaging 2017;30:477-86.

12. Acharya UR, Faust O, Sree SV, Molinari F, Garberoglio R, Suri JS. Cost-effective and non-invasive automated benign and malignant thyroid lesion classification in 3D contrastenhanced ultrasound using combination of wavelets and textures: a class of ThyroScan ${ }^{\mathrm{TM}}$ algorithms. Technol Cancer Res Treat 2011;10:371-80.

13. Ding J, Cheng H, Ning C, Huang J, Zhang Y. Quantitative measurement for thyroid cancer characterization based on 
elastography. J Ultrasound Med 2011;30:1259-66.

14. Acharya UR, Faust O, Sree SV, Molinari F, Suri JS. ThyroScreen system: high resolution ultrasound thyroid image characterization into benign and malignant classes using novel combination of texture and discrete wavelet transform. Comput Methods Programs Biomed 2012;107:233-41.

15. Acharya UR, Vinitha Sree S, Krishnan MM, Molinari F, Garberoglio R, Suri JS. Non-invasive automated 3D thyroid lesion classification in ultrasound: a class of ThyroScan ${ }^{\mathrm{TM}}$ systems. Ultrasonics 2012;52:508-20.

16. Chang Y, Paul AK, Kim N, Baek JH, Choi YJ, Ha EJ, et al. Computer-aided diagnosis for classifying benign versus malignant thyroid nodules based on ultrasound images: a comparison with radiologist-based assessments. Med Phys 2016; 43:554.

17. Prochazka A, Gulati S, Holinka S, Smutek D. Classification of thyroid nodules in ultrasound images using direction-independent features extracted by two-threshold binary decomposition. Technol Cancer Res Treat 2019;18:1533033819830748.

18. Ma J, Wu F, Zhu J, Xu D, Kong D. A pre-trained convolutional neural network based method for thyroid nodule diagnosis. Ultrasonics 2017;73:221-30.

19. Ko SY, Lee JH, Yoon JH, Na H, Hong E, Han K, et al. Deep convolutional neural network for the diagnosis of thyroid nodules on ultrasound. Head Neck 2019;41:885-91.

20. Wang L, Yang S, Yang S, Zhao C, Tian G, Gao Y, et al. Automatic thyroid nodule recognition and diagnosis in ultrasound imaging with the YOLOv2 neural network. World J Surg Oncol 2019;17:12.

21. Karakitsos P, Cochand-Priollet B, Guillausseau PJ, Pouliakis A. Potential of the back propagation neural network in the morphologic examination of thyroid lesions. Anal Quant Cytol Histol 1996;18:494-500.

22. Karakitsos P, Cochand-Priollet B, Pouliakis A, Guillausseau PJ, Ioakim-Liossi A. Learning vector quantizer in the investigation of thyroid lesions. Anal Quant Cytol Histol 1999;21: 201-8.

23. Ippolito AM, De Laurentiis M, La Rosa GL, Eleuteri A, Tagliaferri R, De Placido S, et al. Neural network analysis for evaluating cancer risk in thyroid nodules with an indeterminate diagnosis at aspiration cytology: identification of a lowrisk subgroup. Thyroid 2004;14:1065-71.

24. Shapiro NA, Poloz TL, Shkurupij VA, Tarkov MS, Poloz VV, Demin AV. Application of artificial neural network for classification of thyroid follicular tumors. Anal Quant Cytol Histol 2007;29:87-94.
25. Daskalakis A, Kostopoulos S, Spyridonos P, Glotsos D, Ravazoula P, Kardari M, et al. Design of a multi-classifier system for discriminating benign from malignant thyroid nodules using routinely H\&E-stained cytological images. Comput Biol Med 2008;38:196-203.

26. Varlatzidou A, Pouliakis A, Stamataki M, Meristoudis C, Margari N, Peros G, et al. Cascaded learning vector quantizer neural networks for the discrimination of thyroid lesions. Anal Quant Cytol Histol 2011;33:323-34.

27. Gopinath B, Shanthi N. Development of an automated medical diagnosis system for classifying thyroid tumor cells using multiple classifier fusion. Technol Cancer Res Treat 2015;14: 653-62.

28. Margari N, Mastorakis E, Pouliakis A, Gouloumi AR, Asimis E, Konstantoudakis $\mathrm{S}$, et al. Classification and regression trees for the evaluation of thyroid cytomorphological characteristics: a study based on liquid based cytology specimens from thyroid fine needle aspirations. Diagn Cytopathol 2018;46:670-81.

29. Sanyal P, Mukherjee T, Barui S, Das A, Gangopadhyay P. Artificial intelligence in cytopathology: a neural network to identify papillary carcinoma on thyroid fine-needle aspiration cytology smears. J Pathol Inform 2018;9:43.

30. Liu C, Huang Y, Ozolek JA, Hanna MG, Singh R, Rohde GK. SetSVM: an approach to set classification in nucleibased cancer detection. IEEE J Biomed Health Inform 2019; 23:351-61.

31. Sim I. Two ways of knowing: big data and evidence-based medicine. Ann Intern Med 2016;164:562-3.

32. Mehra S, Tuttle RM, Bergman D, Bernet V, Brett E, Cobin R, et al. Improving the quality of thyroid cancer care: how does the Thyroid Cancer Care Collaborative cross the Institute of Medicine's Quality Chasm? Thyroid 2014;24:615-24.

33. Mehra S, Tuttle RM, Milas M, Orloff L, Bergman D, Bernet $\mathrm{V}$, et al. Database and registry research in thyroid cancer: striving for a new and improved national thyroid cancer database. Thyroid 2015;25:157-68.

34. Toral V, Garcia A, Romero FJ, Morales DP, Castillo E, Parrilla $\mathrm{L}$, et al. Wearable system for biosignal acquisition and monitoring based on reconfigurable technologies. Sensors (Basel) 2019;19:E1590.

35. Lee JE, Lee DH, Oh TJ, Kim KM, Choi SH, Lim S, et al. Clinical feasibility of monitoring resting heart rate using a wearable activity tracker in patients with thyrotoxicosis: prospective longitudinal observational study. JMIR Mhealth Uhealth 2018;6:e159. 
36. Tamura T, Maeda Y, Sekine M, Yoshida M. Wearable photoplethysmographic sensors: past and present. Electronics 2014; 3:282-302.

37. Dooley EE, Golaszewski NM, Bartholomew JB. Estimating accuracy at exercise intensities: a comparative study of selfmonitoring heart rate and physical activity wearable devices. JMIR Mhealth Uhealth 2017;5:e34.
38. Wang R, Blackburn G, Desai M, Phelan D, Gillinov L, Houghtaling P, et al. Accuracy of wrist-worn heart rate monitors. JAMA Cardiol 2017;2:104-6.

39. THYROSCOPE. Detect thyroid disease by your biosignals [Internet]. Scottsdale: THYROSCOPE; c2017 [cited 2019 May 26]. Available from: https://thyroscope.org. 